\title{
ON THE UNIQUE EXTREMALITY OF QUASICONFORMAL MAPPINGS WITH DILATATION BOUNDS
}

\author{
YU-LIANG SHEN
}

(Received March 25, 2002, revised September 5, 2002)

\begin{abstract}
Concerning the problem of extremality of quasiconformal mappings with dilatation bounds, we discuss the unique extremality of the problem and prove the if part of a conjecture on the unique extremality ([G1], [R1]). To this end, we need to investigate a new extremal problem in the infinitesimal setting. In particular, we give a complete description of the unique infinitesimal extremality of partially zero Beltrami differentials.
\end{abstract}

1. Introduction. The problem of extremality of quasiconformal mappings with dilatation bounds has been much investigated in the literature. In this paper, we will discuss the unique extremality of the problem and prove the if part of a conjecture concerning the unique extremality. To make this precise, we state the problem as follows.

Let $R^{\prime}$ and $R$ be two hyperbolic Riemann surfaces covered by the unit disk $\Delta=\{z$ : $|z|<1\}$ in the complex plane. Let a compact, possibly empty, subset $E^{\prime}$ of $R^{\prime}$ be given in such a way that $R^{\prime} \backslash E^{\prime}$ has positive measure, a non-negative measurable function $b(w)$ on $E^{\prime}$ (known as a dilatation bound function) with $\|b\|_{\infty}<1$, and a quasiconformal mapping $F$ of $R^{\prime}$ onto $R$ such that the complex dilatation $\tilde{\mu}$ of $F$ satisfies $|\tilde{\mu}(w)| \leq b(w)$ for a.e. $w \in E^{\prime}$. We denote by $Q\left(F, E^{\prime}, b\right)$ the class of all quasiconformal mappings $G$ of $R^{\prime}$ onto $R$ such that $G$ is homotopic to $F\left(\bmod \partial R^{\prime}\right)$ and that the complex dilatation $\tilde{v}$ of $G$ satisfies $|\tilde{v}(w)| \leq b(w)$ for a.e. $w \in E^{\prime}$. Here $\partial R^{\prime}$ is the ideal boundary of $R^{\prime}$ in the standard sense (see [G2]). Then $F, E^{\prime}$ and $b$ determine the extremal maximal dilatation $K\left(F, E^{\prime}, b\right) \geq 1$, defined as

$$
K\left(F, E^{\prime}, b\right)=\inf \left\{K\left[G \mid R^{\prime} \backslash E^{\prime}\right]: G \in Q\left(F, E^{\prime}, b\right)\right\},
$$

where $K\left[G \mid R^{\prime} \backslash E^{\prime}\right]$ is the maximal dilatation of $G$ on $R^{\prime} \backslash E^{\prime}$. To avoid triviality, we will always assume that $K\left(F, E^{\prime}, b\right)>1$, that is, $Q\left(F, E^{\prime}, b\right)$ contains no mapping which is conformal in $R^{\prime} \backslash E^{\prime}$. An element $G$ of $Q\left(F, E^{\prime}, b\right)$ is called extremal if $K\left[G \mid R^{\prime} \backslash E^{\prime}\right]=$ $K\left(F, E^{\prime}, b\right)$, and uniquely extremal if $K\left[G^{\prime} \mid R^{\prime} \backslash E^{\prime}\right]>K\left[G \mid R^{\prime} \backslash E^{\prime}\right]$ for any other $G^{\prime} \in$ $Q\left(F, E^{\prime}, b\right)$. If $F$ is (uniquely) extremal in $Q\left(F, E^{\prime}, b\right)$, we occasionally say simply that $F$ is (uniquely) extremal.

2000 Mathematics Subject Classifications. Primary 30F60; Secondly 32G15, 30C62, 30C70.

Key words and phrases. Quasiconformal mapping, Beltrami differential, uniquely extremal, uniquely infinitesimally extremal.

Project supported by the National Natural Science Foundation of China and the Natural Science Foundation of Jiangsu Provincial Education Committee. 
As stated above, there have been many literatures on the problem of extremality of quasiconformal mappings with dilatation bounds (see, for example, [F], [FS], [G1], [R1], [Sa15]). Among others, it is known that there always exists at least one extremal mapping in $Q\left(F, E^{\prime}, b\right)$, and a complete characterization of the extremality also appeared in the literature. For our purpose, we recall this precisely as follows.

As usual, we denote by $Q(R)$ the space of all integrable holomorphic quadratic differentials on the surface $R$, and by $M(R)$ the unit ball of the space $L^{\infty}(R)$ of all essentially bounded Beltrami differentials on $R$. Now we let $E_{0}^{\prime}=\left\{w \in E^{\prime}: b(w)=0\right\}$. For the mapping $F$, let $\mu$ denote the complex dilatation of the inverse mapping $f=F^{-1}, k_{F}=\left\|\tilde{\mu} \mid R^{\prime} \backslash E^{\prime}\right\|_{\infty}$, and set

$$
\tau_{F}(z)=\left\{\begin{array}{l}
\mu(z) \quad z \in R \backslash F\left(E^{\prime} \backslash E_{0}^{\prime}\right) \\
k_{F} \mu(z) / b(f(z)) \quad z \in F\left(E^{\prime} \backslash E_{0}^{\prime}\right)
\end{array}\right.
$$

Then we have the following result (see, for example, [Sa4]).

PROPOSITION 1.1. F is extremal if and only if the Beltrami differential $\tau_{F}$ satisfies the condition

$$
\sup \left\{\left|\iint_{R \backslash F\left(E_{0}^{\prime}\right)} \tau_{F} \phi\right| ; \phi \in Q(R),\|\phi\|_{R \backslash F\left(E_{0}^{\prime}\right)}=1\right\}=\left\|\tau_{F}\right\|_{\infty} .
$$

While Proposition 1.1 completely characterizes the extremality of the mapping $F$, less is known for the unique extremality. In several articles (see, for example, [G1], [R1]) it has been pointed out that the unique extremality of $F$ is closely related to the uniqueness of the Hahn-Banach extension of the linear functional $\Lambda_{\tau} \in\left(Q(R) \mid R \backslash F\left(E_{0}^{\prime}\right)\right)^{*}$ induced by $\tau=\tau_{F}, \Lambda_{\tau}(\phi)=\iint_{R \backslash F\left(E_{0}^{\prime}\right)} \tau \phi$. In fact, it was conjectured that $F$ is uniquely extremal if and only if $\Lambda_{\tau}$ has a unique norm-preserving extension to a bounded linear functional from $Q(R) \mid R \backslash F\left(E_{0}^{\prime}\right)$ to $L^{1}\left(R \backslash F\left(E_{0}^{\prime}\right)\right)$. Here $Q(R) \mid R \backslash F\left(E_{0}^{\prime}\right)$ means the restriction to $R \backslash F\left(E_{0}^{\prime}\right)$ of $Q(R)$.

When $E^{\prime}$ is the empty set, the unique extremality has been much discussed recently (see [BLMM], [BMM], [MM], [R4], [Sh1], [Sh2]), and the conjecture was proved affirmatively in [BLMM]. In this paper, we will study the unique extremality for a general set $E^{\prime}$, proving that the if part of the conjecture is still true in this general case.

THEOREM 1.1. Let $F$ be extremal (in the class $Q\left(F, E^{\prime}, b\right)$ ). If $\Lambda_{\tau}\left(\tau=\tau_{F}\right)$ has a unique norm-preserving extension to a bounded linear functional from $Q(R) \mid R \backslash F\left(E_{0}^{\prime}\right)$ to $L^{1}\left(R \backslash F\left(E_{0}^{\prime}\right)\right)$, then $F$ is uniquely extremal.

In order to prove Theorem 1.1, we need to investigate a new extremal problem in the infinitesimal setting, namely, the extremal problem for partially zero Beltrami differentials. In Section 2, we will introduce such an extremal problem and explain how these two extremal problems are related to each other. In Sections 3 and 4, we will give a complete description of the unique infinitesimal extremality of partially zero Beltrami differentials. In Section 5, we 
shall establish a fundamental inequality, which will be used to prove Theorem 1.1 in Section 6.

The author would like to thank the referee for his many valuable suggestions.

2. Partially zero Beltrami differentials. In this section, we will introduce a somewhat new extremal problem in an infinitesimal setting, which, as will be seen, is closely related to the extremal problem of quasiconformal mappings with dilatation bounds. Indeed, in the unit disk case, such an extremal problem has been introduced and dicussed in [SC], where it was used to prove the existence of non-decreasable dilatations in a non-zero infinitesimally equivalent class. For completeness and for generality, we will repeat some discussions from [SC].

Let $E_{0}$, which will be fixed through out Sections 2 through 4, be a compact, possibly empty, subset of $R$ such that $R \backslash E_{0}$ has positive measure, and $\mu_{0} \in L^{\infty}(R)$ be a Beltrami differential which vanishes on the set $E_{0}$. Recall that two elements $\mu$ and $v$ in $L^{\infty}(R)$ are infinitesimally equivalent, denoted by $\mu \approx \nu$, if $\iint_{R} \mu \phi=\iint_{R} \nu \phi$ for all $\phi \in Q(R)$. We denote by $\operatorname{Belt}\left(\mu_{0}\right)$ the set of all elements $\mu$ in $L^{\infty}(R)$ infinitesimally equivalent to $\mu_{0}$ and set

$$
\begin{gathered}
\operatorname{Belt}\left(\mu_{0}, E_{0}\right)=\left\{\mu \in \operatorname{Belt}\left(\mu_{0}\right) ; \mu(z)=0 \text { a.e. } z \in E_{0}\right\}, \\
\left\|\mu_{0}\right\|_{E_{0}}=\inf \left\{\|\mu\|_{\infty} ; \mu \in \operatorname{Belt}\left(\mu_{0}, E_{0}\right)\right\} .
\end{gathered}
$$

An element $\mu \in \operatorname{Belt}\left(\mu_{0}, E_{0}\right)$ is called infinitesimally extremal if $\|\mu\|_{\infty}=\left\|\mu_{0}\right\|_{E_{0}}$, and uniquely infinitesimally extremal if for any other $v \in \operatorname{Belt}\left(\mu_{0}, E_{0}\right),\|v\|_{\infty}>\|\mu\|_{\infty}$. If $\mu_{0}$ is (uniquely) infinitesimally extremal in $\operatorname{Belt}\left(\mu_{0}, E_{0}\right)$, we occasionally say simply that $\mu_{0}$ is (uniquely) infinitesimally extremal.

We then have the following basic result.

THEOREM 2.1. There always exists at least one infinitesimally extremal Beltrami differential in $\operatorname{Belt}\left(\mu_{0}, E_{0}\right)$. Furthermore, if $\operatorname{Belt}\left(\mu_{0}, E_{0}\right)$ contains more than one infinitesimally extremal Beltrami differential, then it must contain infinitely many.

Proof 1. Let $\mu_{n} \in \operatorname{Belt}\left(\mu_{0}, E_{0}\right)$ satisfy $\left\|\mu_{n}\right\|_{\infty} \rightarrow\left\|\mu_{0}\right\|_{E_{0}}$ as $n \rightarrow \infty$. When restricted on $R \backslash E_{0},\left(\mu_{n}\right)$ is a bounded sequence in $L^{\infty}\left(R \backslash E_{0}\right)$. By the *-weak compactness, there exists a subsequence, also denoted by $\left(\mu_{n}\right)$, which converges to a limit $\mu \in L^{\infty}\left(R \backslash E_{0}\right)$ in the *-weak topology, that is, $\iint_{R \backslash E_{0}} \mu_{n} \phi \rightarrow \iint_{R \backslash E_{0}} \mu \phi$ for any $\phi \in L^{1}\left(R \backslash E_{0}\right)$. Now, when $\phi \in Q(R)$, since $\mu_{n} \in \operatorname{Belt}\left(\mu_{0}, E_{0}\right), \iint_{R \backslash E_{0}} \mu_{n} \phi=\iint_{R} \mu_{n} \phi=\iint_{R} \mu_{0} \phi$, we obtain that $\iint_{R \backslash E_{0}} \mu \phi=\iint_{R} \mu_{0} \phi$. Extending $\mu$ to $E_{0}$ be zero, we conclude that $\mu \in$ $\operatorname{Belt}\left(\mu_{0}, E_{0}\right)$. On the other hand, since $\left(\mu_{n}\right)$ converges to $\mu$ in the *-weak topology, $\|\mu\|_{\infty} \leq$ $\lim \inf \left\|\mu_{n}\right\|_{\infty}=\left\|\mu_{0}\right\|_{E_{0}}$, which implies that $\mu \in \operatorname{Belt}\left(\mu_{0}, E_{0}\right)$ is infinitesimally extremal.

Suppose now that $\mu$ and $v$ are two distinct infinitesimally extremal Beltrami differentials in $\operatorname{Belt}\left(\mu_{0}, E_{0}\right)$. For $0<t<1$, set $\mu_{t}=t \mu+(1-t) v$. It is then easy to see that $\mu_{t}$ is infinitesimally extremal in $\operatorname{Belt}\left(\mu_{0}, E_{0}\right)$. 
LEMMA 2.1. For any $\mu \in \operatorname{Belt}\left(\mu_{0}, E_{0}\right)$, it holds that

$$
\sup \left\{\left|\iint_{R \backslash E_{0}} \mu \phi\right| ; \phi \in Q(R),\|\phi\|_{R \backslash E_{0}}=1\right\}=\left\|\mu_{0}\right\|_{E_{0}} .
$$

Proof 2. Let $\mu \in \operatorname{Belt}\left(\mu_{0}, E_{0}\right)$ be given. For any $v \in \operatorname{Belt}\left(\mu_{0}, E_{0}\right)$ and $\phi \in Q(R)$ with $\|\phi\|_{R \backslash E_{0}}=1$, since $\iint_{R \backslash E_{0}} \mu \phi=\iint_{R \backslash E_{0}} \nu \phi$, it follows that $\left|\iint_{R \backslash E_{0}} \mu \phi\right| \leq\|\nu\|_{\infty}$, which implies that

$$
\begin{aligned}
& \sup \left\{\left|\iint_{R \backslash E_{0}} \mu \phi\right| ; \phi \in Q(R),\|\phi\|_{R \backslash E_{0}}=1\right\} \\
& \quad \leq \inf \left\{\|v\|_{\infty} ; v \in \operatorname{Belt}\left(\mu_{0}, E_{0}\right)\right\}=\left\|\mu_{0}\right\|_{E_{0}} .
\end{aligned}
$$

On the other hand, since the set $\left\{\phi \mid R \backslash E_{0} ; \phi \in Q(R)\right\}$ is a closed subspace of $L^{1}\left(R \backslash E_{0}\right)$, by the Hahn-Banach theorem and the Riesz representative theorem, there exists some $v \in$ $L^{\infty}\left(R \backslash E_{0}\right)$ such that

$$
\iint_{R \backslash E_{0}} \mu \phi=\iint_{R \backslash E_{0}} \nu \phi \quad \text { for all } \phi \in Q(R)
$$

and that

$$
\sup \left\{\left|\iint_{R \backslash E_{0}} \mu \phi\right| ; \phi \in Q(R),\|\phi\|_{R \backslash E_{0}}=1\right\}=\|v\|_{\infty} .
$$

Extending $v$ to be zero on $E_{0}$, we obtain from (2.4) that $v \in \operatorname{Belt}\left(\mu_{0}, E_{0}\right)$. Then (2.3) and (2.5) imply that

$$
\sup \left\{\left|\iint_{R \backslash E_{0}} \mu \phi\right| ; \phi \in Q(R),\|\phi\|_{R \backslash E_{0}}=1\right\}=\left\|\mu_{0}\right\|_{E_{0}} .
$$

The proof of Lemma 2.1 also shows that $v \in \operatorname{Belt}\left(\mu_{0}, E_{0}\right)$ is infinitesimally extremal, which gives another proof of the existence part of Theorem 2.1. An immediate consequence of Lemma 2.1 is the following theorem.

THEOREM 2.2. $\mu \in \operatorname{Belt}\left(\mu_{0}, E_{0}\right)$ is infinitesimally extremal if and only if

$$
\sup \left\{\left|\iint_{R \backslash E_{0}} \mu \phi\right| ; \phi \in Q(R),\|\phi\|_{R \backslash E_{0}}=1\right\}=\|\mu\|_{\infty} .
$$

Now, we point out how the two extremal problems are related to each other. Noting Proposition 1.1 and Theorem 2.2, we find that $F$ is extremal in $Q\left(F, E^{\prime}, b\right)$ if and only if the Beltrami differential $\tau_{F}$ is infinitesimally extremal in $\operatorname{Belt}\left(\tau_{F}, F\left(E_{0}^{\prime}\right)\right)$. On the other hand, if $\mu \in \operatorname{Belt}\left(\mu_{0}, E_{0}\right)$ is infinitesimally extremal, then we can conclude by the proof of Lemma 2.1 that $\mu$ is uniquely infinitesimally extremal if and only if the linear functional $\Lambda_{\mu} \in\left(Q(R) \mid R \backslash E_{0}\right)^{*}$ induced by $\mu, \Lambda_{\mu}(\phi)=\iint_{R \backslash E_{0}} \mu \phi$, has a unique norm-preserving extension to a bounded linear functional from $Q(R) \mid R \backslash E_{0}$ to $L^{1}\left(R \backslash E_{0}\right)$. Thus, the conjecture in the Introduction is equivalent to the one that $F$ is uniquely extremal in $Q\left(F, E^{\prime}, b\right)$ if and only if $\tau_{F}$ is uniquely infinitesimally extremal in $\operatorname{Belt}\left(\tau_{F}, F\left(E_{0}^{\prime}\right)\right)$. Hence Theorem 1.1 can be restated as follows. 
THEOREM 2.3. Given the class $Q\left(F, E^{\prime}, b\right)$, if $\tau_{F}$ is uniquely infinitesimally extremal in $\operatorname{Belt}\left(\tau_{F}, F\left(E_{0}^{\prime}\right)\right)$, then $F$ is uniquely extremal.

In order to prove Theorem 2.3, we need to investigate the unique infinitesimal extremality of partially zero Beltrami differentials, which will be done in Sections 3 and 4 . Here we want to discuss a special case. Recall that the boundary dilatation of $\mu_{0}$ is defined to be

$$
b\left(\mu_{0}\right)=\inf \left\{\|\mu \mid R \backslash E\|_{\infty} ; \text { for all } \mu \in \operatorname{Belt}\left(\mu_{0}\right) \text { and compact subsets } E \text { in } R\right\} .
$$

It can be defined equivalently as (see [EGL], [GL])

$$
b\left(\mu_{0}\right)=\sup \left\{\limsup _{n \rightarrow \infty}\left|\iint_{R} \mu_{0} \phi_{n}\right| ; \text { all degenerating sequences }\left(\phi_{n}\right) \text { with }\left\|\phi_{n}\right\| \rightarrow 1\right\} .
$$

Recall that a sequence $\left(\phi_{n}\right)$ in $Q(R)$ is said to be degenerating if $\phi_{n} \rightarrow 0$ locally uniformly in $R$. Clearly, $b\left(\mu_{0}\right) \leq\left\|\mu_{0}\right\|_{E_{0}}$.

THEOREM 2.4. Let $\mu$ be infinitesimally extremal in $\operatorname{Belt}\left(\mu_{0}, E_{0}\right)$. If $b\left(\mu_{0}\right)<\left\|\mu_{0}\right\|_{E_{0}}$, then $\mu$ is uniquely infinitesimally extremal. Furthermore, there exists some element $\phi \in Q(R)$ with $\|\phi\|_{R \backslash E_{0}}=1$ such that $\mu=\|\mu\|_{\infty}|\phi| / \phi \chi_{R \backslash E_{0}}$, where $\chi$ stands for the characteristic function of a set.

Proof 3. Since $\mu$ is infinitesimally extremal, it follows from Theorem 2.2 that there exists a sequence $\left(\phi_{n}\right)$ in $Q(R)$ with $\left\|\phi_{n}\right\|_{R \backslash E_{0}}=1$ such that

$$
\left|\iint_{R \backslash E_{0}} \mu \phi_{n}\right| \rightarrow\|\mu\|_{\infty}
$$

Since $E_{0}$ is compact in $R$, it follows that $\left(\phi_{n}\right)$ is a bounded sequence in $Q(R)$. Otherwise, there would exist some subsequence, also denoted by $\left(\phi_{n}\right)$, such that $\left\|\phi_{n}\right\| \rightarrow \infty$ as $n \rightarrow \infty$. Letting $\tilde{\phi}_{n}=\phi_{n} /\left\|\phi_{n}\right\|$, we obtain $\left\|\tilde{\phi}_{n}\right\|=1$. So there exists a subsequence, still denoted by $\left(\tilde{\phi}_{n}\right)$, such that $\tilde{\phi}_{n}$ tends locally uniformly in $R$ to some $\tilde{\phi}$ in $Q(R)$. By Fatou's Lemma, $\|\tilde{\phi}\| \leq 1$. Then, for any subset $F$ of $R$, noting that $0 \leq\left|\tilde{\phi}_{n}-\tilde{\phi}\right|-\left|\tilde{\phi}_{n}\right|+|\tilde{\phi}| \leq 2|\tilde{\phi}|$, we conclude by Lebesgue's dominated convergence theorem that $\left\|\tilde{\phi_{n}}-\tilde{\phi}\right\|_{F}-\left\|\tilde{\phi_{n}}\right\|_{F}+\|\tilde{\phi}\|_{F} \rightarrow 0$ as $n \rightarrow \infty$. If $F=E_{0}$, then $\left\|\tilde{\phi_{n}}-\tilde{\phi}\right\|_{E_{0}} \rightarrow 0$. On the other hand, $\left\|\tilde{\phi}_{n}\right\|_{E_{0}}=\left\|\phi_{n}\right\|_{E_{0}} /\left\|\phi_{n}\right\|=$ $\left(\left\|\phi_{n}\right\|-1\right) /\left\|\phi_{n}\right\| \rightarrow 1$, so we have $\|\tilde{\phi}\|_{E_{0}}=1$, which contradicts $\|\tilde{\phi}\| \leq 1$.

Now, since $\left(\phi_{n}\right)$ is a bounded sequence in $Q(R)$, there exists a subsequence, also denote by $\left(\phi_{n}\right)$, which converges to some function $\phi \in Q(R)$ locally uniformly in $R$. By the same reasoning as above, we have

$$
\lim _{n \rightarrow \infty}\left(\left\|\phi_{n}-\phi\right\|_{F}-\left\|\phi_{n}\right\|_{F}+\|\phi\|_{F}\right)=0
$$

for any subset $F$ of $R$. In particular, we have

$$
\lim _{n \rightarrow \infty}\left\|\phi_{n}-\phi\right\|_{R \backslash E_{0}}=1-\|\phi\|_{R \backslash E_{0}} .
$$


Under the assumption that $b\left(\mu_{0}\right)<\left\|\mu_{0}\right\|_{E_{0}}$, we want to show that $\|\phi\|_{R \backslash E_{0}}=1$. Suppose to the contrary that $\|\phi\|_{R \backslash E_{0}}<1$, and set

$$
\psi_{n}=\frac{\phi_{n}-\phi}{\left\|\phi_{n}-\phi\right\|_{R \backslash E_{0}}} .
$$

Then $\left(\psi_{n}\right)$ is a sequence in $Q(R)$ which satisfies that $\left\|\psi_{n}\right\|_{R \backslash E_{0}}=1, \psi_{n} \rightarrow 0$ locally uniformly in $R$ and that

$$
\left|\iint_{R \backslash E_{0}} \mu \psi_{n}\right| \rightarrow\|\mu\|_{\infty} .
$$

Since $E_{0}$ is compact in $R,\left\|\psi_{n}\right\| \rightarrow 1$, so (2.11) implies that $b\left(\mu_{0}\right)=b(\mu)=\|\mu\|_{\infty}=$ $\left\|\mu_{0}\right\|_{E_{0}}$. This is a contradiction.

Consequently, $\|\phi\|_{R \backslash E_{0}}=1$, which implies from (2.8) and (2.10) that $\iint_{R \backslash E_{0}} \mu \phi=$ $\|\mu\|_{\infty}$ and hence that $\mu=\|\mu\|_{\infty}|\phi| / \phi \chi_{R \backslash E_{0}}$ as required. Finally, it is easy to see that $\mu$ is uniquely infinitesimally extremal.

REMARK 2.1. For simplicity, we say that a Beltrami differential $\mu_{0}$ which vanishes on the compact set $E_{0}$ is a Strebel differential (with respect to $E_{0}$ ) if $b\left(\mu_{0}\right)<\left\|\mu_{0}\right\|_{E_{0}}$.

3. Characterization of unique infinitesimal extremality. In this section we will characterize the unique infinitesimal extremality of partially zero Beltrami differentials under certain condition. In its proof, we need the following fundamental inequality. Recall that $\mu_{0}$ is a Beltrami differential on $R$ which vanishes on the compact subset $E_{0}$.

LEMMA 3.1. Let $\mu$ and $v$ be two Beltrami differentials in the class $\operatorname{Belt}\left(\mu_{0}, E_{0}\right)$. If $\|\nu\|_{\infty} \leq\|\mu\|_{\infty}$, then

$$
\iint_{R \backslash E_{0}}|\mu-v|^{2}|\phi| \leq 8\|\mu\|_{\infty}\left(\|\mu\|_{\infty}\|\phi\|_{R \backslash E_{0}}-\operatorname{Re} \iint_{R \backslash E_{0}} \mu \phi\right)
$$

for all $\phi \in Q(R)$.

REMARK 3.1. When $E_{0}=\emptyset$, Lemma 3.1 was proved in [BLMM](see also [R2], [R3]), and called the infinitesimal delta inequality. For completeness, we give here a short proof using a discussion from [GL].

Proof of Lemma 3.1. Let $k=\|\mu\|_{\infty}$. For any $\phi \in Q(R), \iint_{R \backslash E_{0}} \mu \phi=$ $\iint_{R \backslash E_{0}} \nu \phi$. Therefore, Lemma 3.1 follows from the following calculation. 


$$
\begin{aligned}
& \iint_{R \backslash E_{0}}|\mu-v|^{2}|\phi|=\iint_{R \backslash E_{0}}\left|\mu-k \frac{|\phi|}{\phi}+k \frac{|\phi|}{\phi}-v\right|^{2}|\phi| \\
& \quad \leq 2 \iint_{R \backslash E_{0}}\left|\mu-k \frac{|\phi|}{\phi}\right|^{2}|\phi|+2 \iint_{R \backslash E_{0}}\left|v-k \frac{|\phi|}{\phi}\right|^{2}|\phi| \\
& \quad=2 \iint_{R \backslash E_{0}}\left(|\mu|^{2}+k^{2}-2 k \operatorname{Re} \mu \frac{\phi}{|\phi|}\right)|\phi|+2 \iint_{R \backslash E_{0}}\left(|\nu|^{2}+k^{2}-2 k \operatorname{Re} v \frac{\phi}{|\phi|}\right)|\phi| \\
& \quad \leq 2 \iint_{R \backslash E_{0}}\left(2 k^{2}|\phi|-2 k \operatorname{Re} \mu \phi\right)+2 \iint_{R \backslash E_{0}}\left(2 k^{2}|\phi|-2 k \operatorname{Re} v \phi\right) \\
& \quad=8 k\left(k \iint_{R \backslash E_{0}}|\phi|-\operatorname{Re} \iint_{R \backslash E_{0}} \mu \phi\right) .
\end{aligned}
$$

For a Beltrami differential $\mu$ in the class Belt $\left(\mu_{0}, E_{0}\right)$, the set $R(\mu)=\{z \in R ;|\mu(z)|=$ $\left.\|\mu\|_{\infty}\right\}$ is called the extremal set for $\mu$. We introduce the Reich's functional $\delta_{\mu}$ on $Q(R) \mid R \backslash E_{0}$ induced by $\mu, \delta_{\mu}(\phi)=\|\mu\|_{\infty}\|\phi\|_{R \backslash E_{0}}-\operatorname{Re} \iint_{R \backslash E_{0}} \mu \phi$. We say that $\mu$ satisfies Reich's condition on a set $E \subset R \backslash E_{0}$ if there exists a sequence $\left(\phi_{n}\right)$ in $Q(R)$ such that $\delta_{\mu}\left(\phi_{n}\right) \rightarrow 0$ and $\liminf \left|\phi_{n}(z)\right|>0$ for almost all $z$ in $E$. We are in a position to prove the main result of this section.

THEOREM 3.1. Let $\mu$ be a Beltrami differential in the class Belt $\left(\mu_{0}, E_{0}\right)$ with $|\mu|=$ $\|\mu\|_{\infty}$ almost everywhere on $R \backslash E_{0}$. Then the following conditions are equivalent:

(a) $\mu$ is uniquely infinitesimally extremal in the class $\operatorname{Belt}\left(\mu_{0}, E_{0}\right)$.

(b) $\mu$ is infinitesimally extremal in the class $\operatorname{Belt}\left(\mu_{0}, E_{0}\right)$ and, for every compact subset $E$ of $R \backslash E_{0}$ with positive measure and every $r>0, \mu \chi_{E}+(1 /(1+r)) \mu \chi_{R \backslash E}$ is a Strebel differential (with respect to $E_{0}$ ).

(c) For every measurable subset $E$ of $R \backslash E_{0}$ with positive measure, there exists a sequence $\left(\phi_{n}\right)$ in $Q(R)$ with $\left\|\phi_{n}\right\|_{R \backslash E_{0}}=1$ such that

$$
\left(\|\mu\|_{\infty}-\operatorname{Re} \iint_{R \backslash E_{0}} \mu \phi_{n}\right) / \iint_{E}\left|\phi_{n}\right| \rightarrow 0 .
$$

(d) $\quad$ s satisfies Reich's condition on $R \backslash E_{0}$.

ProOF 4. Suppose that $\mu$ is uniquely infinitesimally extremal. For every compact subset $E$ of $R \backslash E_{0}$ with positive measure and every $r>0$, let $\mu(r, E)=\mu \chi_{E}+(1 /(1+r)) \mu \chi_{R \backslash E}$. We need to show that $\mu(r, E)$ is a Strebel differential, that is, $b(\mu(r, E))<\|\mu(r, E)\|_{E_{0}}$.

It is easy to see that $b(\mu(r, E)) \leq\|\mu\|_{\infty} /(1+r)$. Suppose to the contrary that $b(\mu(r, E))=\|\mu(r, E)\|_{E_{0}}$. Let $v(r, E)$ be an infinitesimally extremal Beltrami differential in the class $\operatorname{Belt}\left(\mu(r, E), E_{0}\right)$. Then $\|v(r, E)\|_{\infty}=\|\mu(r, E)\|_{E_{0}}=b(\mu(r, E)) \leq\|\mu\|_{\infty} /(1+$ $r)$. Clearly, $v=\mu-\mu(r, E)+v(r, E)=(r /(1+r)) \mu \chi_{R \backslash E}+v(r, E) \in \operatorname{Belt}\left(\mu_{0}, E_{0}\right)$. Since $\|v\|_{\infty} \leq(r /(1+r))\|\mu\|_{\infty}+\|v(r, E)\|_{\infty} \leq\|\mu\|_{\infty}$, we conclude by the unique infinitesimal extremality of $\mu$ that $v=\mu$, that is, $\mu(r, E)=v(r, E)$ is infinitesimally extremal. Since $|\mu|=\|\mu\|_{\infty}$ almost everywhere on $R \backslash E_{0}$, this case cannot occur. So (a) implies (b). 
Now, let $\mu$ satisfies the condition (b). By Theorem 2.4, there exists an element $\phi(r, E)$ in $Q(R)$ with $\|\phi(r, E)\|_{R \backslash E_{0}}=1$ such that the infinitesimally extremal Beltrami differential $v(r, E)$ in $\operatorname{Belt}\left(\mu(r, E), E_{0}\right)$ has the form $\|v(r, E)\|_{\infty}|\phi(r, E)| / \phi(r, E) \chi_{R \backslash E_{0}}$. On the other hand, since $\mu$ is infinitesimally extremal in $\operatorname{Belt}\left(\mu_{0}, E_{0}\right)$, and $v=\mu-\mu(r, E)+v(r, E)=$ $(r /(1+r)) \mu \chi_{R \backslash E}+v(r, E) \in \operatorname{Belt}\left(\mu_{0}, E_{0}\right)$, we have $\|\mu\|_{\infty} \leq\|v\|_{\infty} \leq(r /(1+r))\|\mu\|_{\infty}+$ $\|v(r, E)\|_{\infty}$, so $\|v(r, E)\|_{\infty} \geq\|\mu\|_{\infty} /(1+r)$. Consequently,

$$
\begin{aligned}
\frac{\|\mu\|_{\infty}}{1+r} & \leq\|v(r, E)\|_{\infty}=\operatorname{Re} \iint_{R \backslash E_{0}} v(r, E) \phi(r, E) \\
& =\operatorname{Re} \iint_{R \backslash E_{0}} \mu(r, E) \phi(r, E)=\operatorname{Re} \iint_{E} \mu \phi(r, E)+\operatorname{Re} \frac{1}{1+r} \iint_{R \backslash E_{0} \backslash E} \mu \phi(r, E) .
\end{aligned}
$$

Thus,

$$
\|\mu\|_{\infty}-\operatorname{Re} \iint_{R \backslash E_{0}} \mu \phi(r, E)=\operatorname{Re} \iint_{E} r \mu \phi(r, E) \leq\|\mu\|_{\infty} r \iint_{E}|\phi(r, E)| .
$$

For each measurable subset $E$ of $R$ with positive measure, choose a compact subset $\tilde{E}$ of $E$ with positive measure. Then for any $r>0$, there exists an element $\phi(r, \tilde{E})$ in $Q(R)$ with $\|\phi(r, \tilde{E})\|_{R \backslash E_{0}}=1$ such that

$$
\|\mu\|_{\infty}-\operatorname{Re} \iint_{R \backslash E_{0}} \mu \phi(r, \tilde{E}) \leq\|\mu\|_{\infty} r \iint_{\tilde{E}}|\phi(r, \tilde{E})| \leq\|\mu\|_{\infty} r \iint_{E}|\phi(r, \tilde{E})| .
$$

For $n \geq 1$, set $r=1 / n$ and $\phi_{n}=\phi(r, \tilde{E})$. Then we conclude by (3.3) that

$$
0 \leq\left(\|\mu\|_{\infty}-\operatorname{Re} \iint_{R \backslash E_{0}} \mu \phi_{n}\right) / \iint_{E}\left|\phi_{n}\right| \leq \frac{\|\mu\|_{\infty}}{n} \rightarrow 0 .
$$

So (b) implies (c).

Finally, let the condition (c) be satisfied. Suppose that $\mu$ is not uniquely infinitesimally extremal. Then there would exist some $v$ in the class $\operatorname{Belt}\left(\mu_{0}, E_{0}\right)$ such that $\|v\|_{\infty} \leq\|\mu\|_{\infty}$ and that $|v-\mu| \geq \varepsilon_{0}>0$ on some positive measure subset $E$ of $R \backslash E_{0}$. Note that for this set $E$, there exists a sequence $\left(\phi_{n}\right)$ in $Q(R)$ with $\left\|\phi_{n}\right\|_{R \backslash E_{0}}=1$ such that

$$
\left(\|\mu\|_{\infty}-\operatorname{Re} \iint_{R \backslash E_{0}} \mu \phi_{n}\right) / \iint_{E}\left|\phi_{n}\right| \rightarrow 0 .
$$

On the other hand, by Lemma 3.1 we have that

$$
\varepsilon_{0}^{2} \iint_{E}\left|\phi_{n}\right| \leq \iint_{R \backslash E_{0}}|v-\mu|^{2}\left|\phi_{n}\right| \leq C\|\mu\|_{\infty}\left(\|\mu\|_{\infty}-\operatorname{Re} \iint_{R \backslash E_{0}} \mu \phi_{n}\right),
$$

which contradicts (3.4). So (c) implies (a).

We will prove the equivalence of (a) and (d) in the next section (Theorem 4.1).

REMARK 3.2. From the proof we see that it holds that $(\mathrm{b}) \Rightarrow(\mathrm{c}) \Rightarrow$ (a) for any Beltrami differential $\mu$ in the class $\operatorname{Belt}\left(\mu_{0}, E_{0}\right)$. The condition $|\mu|=\|\mu\|_{\infty}$ almost everywhere in $R \backslash E_{0}$ is only used in the proof of $(\mathrm{a}) \Rightarrow(\mathrm{b})$. Indeed, when $\mu$ satisfies the condition (a) (without the condition that $|\mu|=\|\mu\|_{\infty}$ almost everywhere in $R \backslash E_{0}$ ), (b) still holds for 
those compact subsets $E$ of $R \backslash E_{0}$ with positive measure and $\|\mu \mid E\|_{\infty}=\|\mu\|_{\infty}$. We will use this fact in the next section.

REMARK 3.3. We say that $\mu$ is uniquely infinitesimally extremal with respect to $S \subset$ $R \backslash E_{0}$ with positive measure if for any other $v \in \operatorname{Belt}\left(\mu_{0}, E_{0}\right)$ with $\|v\|_{\infty} \leq\|\mu\|_{\infty}, \mu=v$ almost everywhere on $S$. Examining the proof of $(c) \Rightarrow(a)$, we find that if the condition (c) is satisfied for every compact subset $E$ of $S \subset R \backslash E_{0}$ with positive measure, then $\mu$ is uniquely infinitesimally extremal with respect to $S$.

4. Characterization of unique infinitesimal extremality (continued). We continue to discuss the unique infinitesimal extremality of partially zero Beltrami differentials. We will modify the discussion in [BLMM].

In general, for a bounded linear functional $\Lambda$ with real part $\lambda$ on a subspace $Y$ of a normed space $X$, we may define

$$
\bar{\lambda}\left(x_{0}\right)=\inf _{y \in Y}\left\{\lambda(y)+\|\lambda\|\left\|y-x_{0}\right\|\right\}
$$

and

$$
\underline{\lambda}\left(x_{0}\right)=\sup _{y \in Y}\left\{\lambda(y)-\|\lambda\|\left\|y-x_{0}\right\|\right\} .
$$

The analysis in the proof of the Hahn-Banach theorem leads to the following lemma.

LEMMA 4.1. $\Lambda$ has a unique norm-preserving extension from $Y$ to $X$ if and only if $\bar{\lambda}\left(x_{0}\right)=\underline{\lambda}\left(x_{0}\right)$ for all $x_{0} \in X \backslash Y$.

We say that $\lambda$ satisfies the unique approximation property at $x_{0} \in X \backslash Y$ if there exists sequences $\left(y_{n 1}\right)$ and $\left(y_{n 2}\right)$ in $Y$ such that

$$
\lambda\left(y_{n 1}-y_{n 2}\right)=\|\lambda\|\left(\left\|y_{n 1}-x_{0}\right\|+\left\|y_{n 2}-x_{0}\right\|\right)+o(1) .
$$

Then we have

LEMMA 4.2. $\Lambda$ has a unique norm-preserving extension from $Y$ to $X$ if and only if $\lambda$ satisfies the unique approximation property at each $x_{0} \in X \backslash Y$.

We now proceed to discuss the unique infinitesimal extremality of a Beltrami differential. Let, as before, $\mu_{0}$ be a Beltrami differential on $R$ which is zero on the compact subset $E_{0}$. Then we have

LEMmA 4.3. If $\mu \in \operatorname{Belt}\left(\mu_{0}, E_{0}\right)$ satisfies Reich's condition on a set $E \subset R \backslash E_{0}$, then $\mu$ is uniquely infinitesimally extremal in the class $\operatorname{Belt}\left(\mu_{0}, E_{0}\right)$ with respect to $E$.

Proof 5. Suppose that $\mu$ is not uniquely infinitesimally extremal with respect to $E$. Then there would exist some $v$ in the class $\operatorname{Belt}\left(\mu_{0}, E_{0}\right)$ such that $\|v\|_{\infty} \leq\|\mu\|_{\infty}$ and that $|v-\mu| \geq \varepsilon_{0}>0$ on some positive measure subset $\tilde{E}$ of $E$. It follows from Lemma 3.1 that

$$
\varepsilon_{0}^{2} \iint_{\tilde{E}}|\phi| \leq \iint_{R \backslash E_{0}}|\nu-\mu|^{2}|\phi| \leq C\|\mu\|_{\infty} \delta_{\mu}(\phi)
$$


for all $\phi \in Q(R)$.

On the other hand, since $\mu$ satisfies Reich's condition on $E$, there exists a sequence $\left(\phi_{n}\right)$ in $Q(R)$ such that $\lim \inf \left|\phi_{n}\right|>0$ almost everywhere in $E$ and that $\delta_{\mu}\left(\phi_{n}\right) \rightarrow 0$. Applying Fatou's Lemma, we then obtain a contradiction from (4.1).

LEMMA 4.4. If $\mu$ is uniquely infinitesimally extremal in the class $\operatorname{Belt}\left(\mu_{0}, E_{0}\right)$, then $\mu$ satisfies Reich's condition on its extremal set.

Proof 6. Suppose that $\mu$ is uniquely infinitesimally extremal in the class $\operatorname{Belt}\left(\mu_{0}, E_{0}\right)$, and let $E=R(\mu)$ be its extremal set. Without loss of generality, we may assume that $\|\mu\|_{\infty}=1$. Take $\phi \in Q(R)$ such that $\|\phi\|_{R \backslash E_{0}}=1$, and let $\psi=|\phi| \bar{\mu} \chi_{E}$. Clearly, $\psi \in L^{1}\left(R \backslash E_{0}\right)$ and

$$
\iint_{R \backslash E_{0}} \mu \psi=\iint_{E}|\phi|=\|\psi\|_{R \backslash E_{0}} .
$$

Since $\mu$ is uniquely infinitesimally extremal in $\operatorname{Belt}\left(\mu_{0}, E_{0}\right), \Lambda_{\mu}(\psi)=\iint_{R \backslash E_{0}} \mu \psi$ is the unique norm-preserving extension from $Q(R) \mid R \backslash E_{0}$ to $L^{1}\left(R \backslash E_{0}\right)$. By Lemma 4.1, for the real part $\lambda_{\mu}=\operatorname{Re} \Lambda_{\mu}$, noting that $\left\|\lambda_{\mu}\right\|=\left\|\Lambda_{\mu}\right\|=\|\mu\|_{\infty}=1$, there exists a sequence $\left(\phi_{n}\right)$ in $Q(R)$ such that $\lambda_{\mu}\left(\phi_{n}\right)-\left\|\phi_{n}-\psi\right\|_{R \backslash E_{0}} \rightarrow \lambda_{\mu}(\psi)$, that is,

$$
\operatorname{Re} \iint_{R \backslash E_{0}} \mu \psi+\left\|\phi_{n}-\psi\right\|_{R \backslash E_{0}}-\operatorname{Re} \iint_{R \backslash E_{0}} \mu \phi_{n} \rightarrow 0 .
$$

Consequently,

$$
\begin{aligned}
0 & \leq \delta_{\mu}\left(\phi_{n}\right)=\left\|\phi_{n}\right\|_{R \backslash E_{0}}-\operatorname{Re} \iint_{R \backslash E_{0}} \mu \phi_{n} \\
& \leq\|\psi\|_{R \backslash E_{0}}+\left\|\phi_{n}-\psi\right\|_{R \backslash E_{0}}-\operatorname{Re} \iint_{R \backslash E_{0}} \mu \phi_{n} \\
& =\operatorname{Re} \iint_{R \backslash E_{0}} \mu \psi+\left\|\phi_{n}-\psi\right\|_{R \backslash E_{0}}-\operatorname{Re} \iint_{R \backslash E_{0}} \mu \phi_{n} \rightarrow 0 .
\end{aligned}
$$

On the other hand, since

$$
\begin{aligned}
0 & \leq \iint_{E}\left(|\psi|+\left|\phi_{n}-\psi\right|-\left|\phi_{n}\right|\right) \\
& \leq\|\psi\|_{R \backslash E_{0}}+\left\|\phi_{n}-\psi\right\|_{R \backslash E_{0}}-\left\|\phi_{n}\right\|_{R \backslash E_{0}} \\
& \leq\|\psi\|_{R \backslash E_{0}}+\left\|\phi_{n}-\psi\right\|_{R \backslash E_{0}}-\operatorname{Re} \iint_{R \backslash E_{0}} \mu \phi_{n} \rightarrow 0,
\end{aligned}
$$

we may assume without loss of generality that $|\psi|+\left|\phi_{n}-\psi\right|-\left|\phi_{n}\right| \rightarrow 0$ for almost all $z \in E$. Hence $\liminf \left|\phi_{n}(z)\right| \geq|\psi(z)|=|\phi(z)|>0$ for almost all $z \in E$.

An immediate consequence of Lemmas 4.3 and 4.4 is the following theorem, which gives another characterization of the unique infinitesimal extremality of Beltrami differentials with constant absolute value on $R \backslash E_{0}$ (see Theorem 3.1). 
THEOREM 4.1. If $\mu \in \operatorname{Belt}\left(\mu_{0}, E_{0}\right)$ satisfies the condition that $|\mu|=\|\mu\|_{\infty}$ almost everywhere in $R \backslash E_{0}$, then $\mu$ is uniquely infinitesimally extremal in $\operatorname{Belt}\left(\mu_{0}, E_{0}\right)$ if and only if $\mu$ satisfies Reich's condition on $R \backslash E_{0}$.

We are now in a position to characterize the unique infinitesimal extremality for an arbitrary Beltrami differential. Let $\mu \in \operatorname{Belt}\left(\mu_{0}, E_{0}\right)$. We say that a Beltrami differential $\eta$ is an admissible variation of $\mu$ if $\eta$ equals zero on $E_{0},\|\eta\|_{\infty} \leq\|\mu\|_{\infty}$, and if there exists some, possibly empty, subset $E$ of $R \backslash E_{0}$ such that $|\mu| \leq k_{0}<k=\|\mu\|_{\infty}$ almost everywhere in $E$ and $\mu=\eta$ in $R \backslash E$.

LEMMA 4.5. If $\mu$ is (uniquely) infinitesimally extremal in the class $\operatorname{Belt}\left(\mu_{0}, E_{0}\right)$, then every admissible variation $\eta$ of $\mu$ is (uniquely) infinitesimally extremal.

Proof 7. Suppose $\mu$ is infinitesimally extremal in the class $\operatorname{Belt}\left(\mu_{0}, E_{0}\right)$, and $\eta$ is any admissible variation of $\mu$. Then there exists a subset $E$ of $R \backslash E_{0}$ such that $|\mu| \leq k_{0}<k=$ $\|\mu\|_{\infty}$ almost everywhere in $E$ and $\mu=\eta$ in $R \backslash E$. Take any real number $t>2 k /\left(k-k_{0}\right)$. Then, for any Beltrami differential $\eta^{\prime}$ in the class Belt $\left(\eta, E_{0}\right), t \mu+\eta^{\prime}-\eta \in \operatorname{Belt}\left(t \mu, E_{0}\right)$. Since $t \mu$ is infinitesimally extremal, we have

$$
\begin{aligned}
t k & =\|t \mu\|_{\infty} \leq\left\|\eta^{\prime}\right\|_{\infty}+\|t \mu-\eta\|_{\infty} \\
& \leq\left\|\eta^{\prime}\right\|_{\infty}+\max \left\{(t-1) k, t k_{0}+k\right\}=\left\|\eta^{\prime}\right\|_{\infty}+(t-1) k,
\end{aligned}
$$

so $\left\|\eta^{\prime}\right\|_{\infty} \geq k \geq\|\eta\|_{\infty}$, that is, $\eta$ is infinitesimally extremal.

If $\mu$ is uniquely infinitesimally extremal in the class $\operatorname{Belt}\left(\mu_{0}, E_{0}\right)$, the above reasoning also shows that $\eta$ is uniquely infinitesimally extremal.

Now we can prove the following general characterization theorem for the unique infinitesimal extremality of Beltrami differentials.

THEOREM 4.2. Let $\mu \in \operatorname{Belt}\left(\mu_{0}, E_{0}\right)$. The following conditions are equivalent:

(1) $\mu$ is uniquely infinitesimally extremal in $\operatorname{Belt}\left(\mu_{0}, E_{0}\right)$.

(2) $\mu$ is infinitesimally extremal in $\operatorname{Belt}\left(\mu_{0}, E_{0}\right)$ and, for every $r>0$, every admissible variation $\eta$ of $\mu$, and every compact subset $E$ of $R(\eta)$ with positive measure, $\eta \chi_{E}+$ $(1 /(1+r)) \eta \chi_{R \backslash E}$ is a Strebel differential.

(3) For every admissible variation $\eta$ of $\mu$ and every compact subset $E$ of $R(\eta)$ with positive measure, there exists a sequence $\left(\phi_{n}\right)$ in $Q(R)$ with $\left\|\phi_{n}\right\|_{R \backslash E_{0}}=1$ such that

$$
\left(\|\eta\|_{\infty}-\operatorname{Re} \iint_{R \backslash E_{0}} \eta \phi_{n}\right) / \iint_{E}\left|\phi_{n}\right| \rightarrow 0 .
$$

(4) Every admissible variation $\eta$ of $\mu$ is uniquely infinitesimally extremal with respect to $R(\eta)$.

(5) Every admissible variation $\eta$ of $\mu$ is uniquely infinitesimally extremal.

(6) Every admissible variation $\eta$ of $\mu$ satisfies reich's condition on $R(\eta)$.

(7) $\lambda_{\mu}$ satisfies the unique approximation property at each $\psi \in L^{1}\left(R \backslash E_{0}\right) \backslash Q(R) \mid R \backslash$ $E_{0}$. 
ProOF 8. (1) is equivalent to (7) by Lemma 4.2.

$(1) \Rightarrow(2)$. Since $\mu$ is uniquely infinitesimally extremal, it is definitely infinitesimally extremal, and every admissible variation $\eta$ of $\mu$ is also uniquely infinitesimally extremal by Lemma 4.5. Hence (2) can be proved by the same method as in the proof of $(a) \Rightarrow(b)$ of Theorem 3.1 (see remark 3.2).

(2) $\Rightarrow$ (3). Since $\mu$ is infinitesimally extremal, every admissible variation $\eta$ of $\mu$ is also infinitesimally extremal by Lemma 4.5. Therefore (3) can be proved by the same method as in the proof of $(b) \Rightarrow(c)$ of Theorem 3.1.

$(3) \Rightarrow(4)$. This can be proved by the same method as in the proof of $(c) \Rightarrow(a)$ of Theorem 3.1(see remark 3.3).

(4) $\Rightarrow$ (1). Suppose that $\mu$ is not uniquely infinitesimally extremal. Then there would exist some $v$ in the class $\operatorname{Belt}\left(\mu_{0}, E_{0}\right)$ such that $\|v\|_{\infty} \leq\|\mu\|_{\infty}$ and that $|\nu-\mu| \geq \varepsilon_{0}>0$ on some positive measure compact subset $E$ of $R \backslash E_{0}$. Noting that $\mu$ itself is an admissible variation of $\mu$, we conclude that the set $\left\{z \in E ;|\mu(z)|=\|\mu\|_{\infty}\right\}$ must be a set of measure zero. So we may assume that for some $k_{0}<k=\|\mu\|_{\infty}$, the set $\tilde{E}=\left\{z \in E:|\mu(z)| \leq k_{0}\right\}$ is compact and has positive measure.

Define $\eta=k((\mu-v) /|\mu-\nu|) \chi_{\tilde{E}}+\mu \chi_{R \backslash \tilde{E}}$. Then $\eta$ is an admissible variation of $\mu$. Now set $\eta^{\prime}=\eta+v-\mu$. Then $\eta^{\prime} \in \operatorname{Belt}\left(\eta, E_{0}\right)$. Noting that

$$
\eta^{\prime}=k \frac{\mu-v}{|\mu-v|} \chi_{\tilde{E}}+\mu \chi_{R \backslash \tilde{E}}+v-\mu=\left(\frac{k}{|\mu-v|}-1\right)(\mu-v) \chi_{\tilde{E}}+v \chi_{R \backslash \tilde{E}},
$$

we get $\left|\eta^{\prime}\right| \leq \max \{|k-| \mu-\nu||, k\}=k$, which implies that $\left\|\eta^{\prime}\right\|_{\infty} \leq k$. Since $|\eta|=k$ on $\tilde{E}$, and $\eta$ is uniquely infinitesimally extremal with respect to its extremal subset $R(\eta)$, we conclude that $\eta^{\prime}=\eta$ and consequently that $\mu=v$ on $\tilde{E} \subset E$, which is a contradiction.

$(1) \Rightarrow(5)$. This follows directly from Lemma 4.5 .

$(5) \Rightarrow(6)$. This follows directly from Lemma 4.4 .

$(6) \Rightarrow(4)$. This follows directly from Lemma 4.3 .

5. A fundamental inequality. In this section, we establish a fundamental inequality parallel to the delta inequality in [BLMM], which will be used to prove Theorem 2.3 in the next section. We shall repeat some discussion from [BLMM] for completeness.

LEMMA 5.1. Let $G$ be a quasiconformal mapping in the class $Q\left(F, E^{\prime}, b\right)$. Let, as before, $\tilde{\mu}, \tilde{v}, \mu$ and $v$ denote the complex dilatations of the mappings $F, G, f=F^{-1}$ and $g=G^{-1}$, respectively. If $k_{G} \leq k_{F}$, then

$$
\iint_{R \backslash F\left(E_{0}^{\prime}\right)}\left|\frac{\tilde{\mu}(f)-\tilde{v}(f)}{1-\overline{\tilde{\mu}(f)} \tilde{v}(f)}\right|^{2}|\phi| \leq C\left(k_{F}\|\phi\|_{R \backslash F\left(E_{0}^{\prime}\right)}-\operatorname{Re} \iint_{R \backslash F\left(E_{0}^{\prime}\right)} \tau_{F} \phi\right),
$$

for all $\phi \in Q(R)$. The constant $C$ depends only on $k_{F}$ and $\|b\|_{\infty}$. 
Proof 9. Set $\tau=\tau_{F}, k=k_{F}, \alpha=\tilde{\mu}(f), \beta=\tilde{v}(f)$. Since $G \in Q\left(F, E^{\prime}, b\right)$, it follows from the main inequality of Reich-Strebel (see [G2]) that

$$
\iint_{R}|\phi| \leq \iint_{R}|\phi| \frac{|1-\mu \phi /| \phi||^{2}}{1-|\mu|^{2}} \frac{|1+\beta(\mu / \alpha)(\phi /|\phi|)(1-\bar{\mu} \bar{\phi} /|\phi|) /(1-\mu \phi /|\phi|)|^{2}}{1-|\beta|^{2}},
$$

for all $\phi \in Q(R)$, or equivalently (see [R2], [R3]),

$$
\operatorname{Re} \iint_{R} \frac{(\alpha-\beta)(1-\alpha \bar{\beta})}{\left(1-|\alpha|^{2}\right)\left(1-|\beta|^{2}\right)} \frac{\mu}{\alpha} \phi \leq \iint_{R} \frac{|\alpha-\beta|^{2}}{\left(1-|\alpha|^{2}\right)\left(1-|\beta|^{2}\right)}|\phi| .
$$

Noting that $\mu / \alpha=-\overline{\partial f} / \partial f$ and $\alpha=\beta=0$ on $R \backslash F\left(E_{0}^{\prime}\right)$, we obtain from (5.1) that

$$
\operatorname{Re} \iint_{R \backslash F\left(E_{0}^{\prime}\right)} \frac{(\alpha-\beta)(1-\alpha \bar{\beta})}{\left(1-|\alpha|^{2}\right)\left(1-|\beta|^{2}\right)} \frac{\mu}{\alpha} \phi \leq \iint_{R \backslash F\left(E_{0}^{\prime}\right)} \frac{|\alpha-\beta|^{2}}{\left(1-|\alpha|^{2}\right)\left(1-|\beta|^{2}\right)}|\phi| .
$$

Adding $\operatorname{Re} \iint_{R \backslash F\left(E_{0}^{\prime}\right)}\left((\beta-\alpha)(1-\alpha \bar{\beta}) /\left(1-|\alpha|^{2}\right)\left(1-|\beta|^{2}\right)\right)(|\alpha| / \alpha)|\phi|$ to both sides of the above inequality, we conclude that

$$
\begin{aligned}
\iint_{R \backslash F\left(E_{0}^{\prime}\right)} & \frac{(1-|\alpha|)^{2}|\alpha-\beta|^{2}+\left(1-|\alpha|^{2}\right)\left(|\alpha|^{2}-|\beta|^{2}\right)}{2|\alpha|\left(1-|\alpha|^{2}\right)\left(1-|\beta|^{2}\right)}|\phi| \\
& \leq \operatorname{Re} \iint_{R \backslash F\left(E_{0}^{\prime}\right)} \frac{(\alpha-\beta)(1-\alpha \bar{\beta})}{\left(1-|\alpha|^{2}\right)\left(1-|\beta|^{2}\right)} \frac{1}{\alpha}(|\mu||\phi|-\mu \phi),
\end{aligned}
$$

or equivalently,

$$
\begin{aligned}
& \iint_{R \backslash F\left(E_{0}^{\prime}\right)} \frac{(1-|\alpha|)|\alpha-\beta|^{2}}{2|\alpha|(1+|\alpha|)\left(1-|\beta|^{2}\right)}|\phi| \\
& \quad \leq \operatorname{Re} \iint_{R \backslash F\left(E_{0}^{\prime}\right)} \frac{(\alpha-\beta)(1-\alpha \bar{\beta})}{\left(1-|\alpha|^{2}\right)\left(1-|\beta|^{2}\right)} \frac{1}{\alpha}(|\mu||\phi|-\mu \phi) \\
& \quad+\iint_{R \backslash F\left(E_{0}^{\prime}\right)} \frac{|\beta|^{2}-|\alpha|^{2}}{2|\alpha|\left(1-|\beta|^{2}\right)}|\phi| .
\end{aligned}
$$

We first assume that $|\tau| \geq k / 2$ on $R \backslash F\left(E_{0}^{\prime}\right)$, that is, $|\alpha|=|\mu| \geq k / 2$ on $R \backslash F\left(E^{\prime}\right)$ and $|\alpha|=|\mu| \geq b(f) / 2$ on $F\left(E^{\prime} \backslash E_{0}^{\prime}\right)$. Note that on $R \backslash F\left(E^{\prime}\right)$

$$
\frac{(1-|\alpha|)|\alpha-\beta|^{2}}{2|\alpha|(1+|\alpha|)\left(1-|\beta|^{2}\right)}|\phi| \geq \frac{\left(1-\max \left\{k,\|b\|_{\infty}\right\}\right)|\alpha-\beta|^{2}}{4}|\phi| .
$$

Using the identity

$$
|| w|-w|^{2}=2|w|(|w|-\operatorname{Re} w)
$$

we get 


$$
\begin{aligned}
& \operatorname{Re} \frac{(\alpha-\beta)(1-\alpha \bar{\beta})}{\left(1-|\alpha|^{2}\right)\left(1-|\beta|^{2}\right)} \frac{1}{\alpha}(|\mu||\phi|-\mu \phi) \\
& \quad \leq \frac{|\alpha-\beta||1-\alpha \bar{\beta}|}{\left(1-|\alpha|^{2}\right)\left(1-|\beta|^{2}\right)} \frac{1}{|\alpha|}\{2|\mu||\phi|(|\mu||\phi|-\operatorname{Re} \mu \phi)\}^{1 / 2} \\
& \quad \leq \begin{cases}4 /\left(1-\|b\|_{\infty}^{2}\right)^{2} \sqrt{1 / k} \sqrt{|\alpha-\beta|^{2}|\phi|} \sqrt{k|\phi|-\operatorname{Re} \tau \phi} \quad \text { in } F\left(E^{\prime} \backslash E_{0}^{\prime}\right) \\
4 /\left(1-k^{2}\right)^{2} \sqrt{1 / k} \sqrt{|\alpha-\beta|^{2}|\phi|} \sqrt{k|\phi|-\operatorname{Re} \tau \phi} \quad \text { in } R \backslash F\left(E^{\prime}\right)\end{cases} \\
& \quad \leq \frac{4}{\sqrt{k}\left(1-\left(\max \left\{k,\|b\|_{\infty}\right\}\right)^{2}\right)^{2}} \sqrt{|\alpha-\beta|^{2}|\phi|} \sqrt{k|\phi|-\operatorname{Re} \tau \phi} .
\end{aligned}
$$

We also have

$$
\begin{aligned}
\frac{|\beta|^{2}-|\alpha|^{2}}{2|\alpha|\left(1-|\beta|^{2}\right)}|\phi| & \leq\left\{\begin{array}{l}
2 /\left(k\left(1-\|b\|_{\infty}^{2}\right)\right)(k|\phi|-\operatorname{Re} \tau \phi) \quad \text { in } F\left(E^{\prime} \backslash E_{0}^{\prime}\right) \\
2 /\left(k\left(1-k^{2}\right)\right)(k|\phi|-\operatorname{Re} \tau \phi) \quad \text { in } R \backslash F\left(E^{\prime}\right)
\end{array}\right. \\
& \leq \frac{2}{k\left(1-\left(\max \left\{k,\|b\|_{\infty}\right\}\right)^{2}\right)}(k|\phi|-\operatorname{Re} \tau \phi) .
\end{aligned}
$$

It follows from (5.3) through (5.6) that

$$
\begin{aligned}
& \iint_{R \backslash F\left(E_{0}^{\prime}\right)}|\alpha-\beta|^{2}|\phi| \\
& \leq C_{1}\left(k,\|b\|_{\infty}\right)\left(\iint_{R \backslash F\left(E_{0}^{\prime}\right)} \sqrt{|\alpha-\beta|^{2}|\phi|} \sqrt{k|\phi|-\operatorname{Re} \tau \phi}+\iint_{R \backslash F\left(E_{0}^{\prime}\right)}(k|\phi|-\operatorname{Re} \tau \phi)\right),
\end{aligned}
$$

where

$$
C_{1}\left(k,\|b\|_{\infty}\right)=\frac{32}{k\left(1-\left(\max \left\{k,\|b\|_{\infty}\right\}\right)^{2}\right)^{3}} .
$$

Using the Cauchy-Schwartz inequality, we obtain from (5.7) that

$$
\begin{aligned}
& \iint_{R \backslash F\left(E_{0}^{\prime}\right)} \mid \alpha-\left.\beta\right|^{2}|\phi| \leq C_{1}\left(k,\|b\|_{\infty}\right) \\
& \times\left(\sqrt{\iint_{R \backslash F\left(E_{0}^{\prime}\right)}|\alpha-\beta|^{2}|\phi| \iint_{R \backslash F\left(E_{0}^{\prime}\right)}(k|\phi|-\operatorname{Re} \tau \phi)}\right. \\
&\left.+\iint_{R \backslash F\left(E_{0}^{\prime}\right)}(k|\phi|-\operatorname{Re} \tau \phi)\right) .
\end{aligned}
$$

By (5.8) it sufficies to show the lemma assuming that $\iint_{R \backslash F\left(E_{0}^{\prime}\right)}(k|\phi|-\operatorname{Re} \tau \phi) \neq 0$. Letting

$$
t^{2}=\iint_{R \backslash F\left(E_{0}^{\prime}\right)}|\alpha-\beta|^{2}|\phi| / \iint_{R \backslash F\left(E_{0}^{\prime}\right)}(k|\phi|-\operatorname{Re} \tau \phi),
$$


(5.8) then implies that $t \leq C_{1}\left(k,\|b\|_{\infty}\right)(1+1 / t)$, which implies that $t$ is bounded, so

$$
\iint_{R \backslash F\left(E_{0}^{\prime}\right)}|\alpha-\beta|^{2}|\phi| \leq C_{2}\left(k,\|b\|_{\infty}\right) \iint_{R \backslash F\left(E_{0}^{\prime}\right)}(k|\phi|-\operatorname{Re} \tau \phi) .
$$

Consequently,

$$
\iint_{R \backslash F\left(E_{0}^{\prime}\right)}\left|\frac{\alpha-\beta}{1-\bar{\alpha} \beta}\right|^{2}|\phi| \leq C_{3}\left(k,\|b\|_{\infty}\right) \iint_{R \backslash F\left(E_{0}^{\prime}\right)}(k|\phi|-\operatorname{Re} \tau \phi) .
$$

Now we suppose that the set $E=\left\{z \in R \backslash F\left(E_{0}^{\prime}\right) ;|\tau|<k / 2\right\}$ has positive measure. Choose some non-zero element $\psi \in Q\left(R^{\prime}\right)$. We define a Beltrami differential $\tilde{\eta}$ on $R^{\prime}$ as follows: When $w \in R^{\prime} \backslash f(E), \tilde{\eta}(w)=0$. In the case when $w \in f(E) \cap\left(R^{\prime} \backslash E^{\prime}\right)$, if $\tilde{\mu}(w) \neq \tilde{v}(w)$, then $\tilde{\eta}(w)$ is the unique point $\zeta$ on the hyperbolic circle $\rho(\zeta, \tilde{\mu}(w))=$ $\rho(0, k / 2)$ whose hyperbolic distance to $\tilde{v}(w)$ is a minimal; if $\tilde{\mu}(w)=\tilde{v}(w) \neq 0$, then $\tilde{\eta}(w)=a \tilde{\mu}(w)$, where $a$ is a positive constant, such that $\tilde{\eta}(w)$ is on the hyperbolic circle $\rho(\zeta, \tilde{\mu}(w))=\rho(0, k / 2)$; if $\tilde{\mu}(w)=\tilde{v}(w)=0$, then $\tilde{\eta}(w)=k|\psi(w)| /(2 \psi(w))$. In the case when $w \in f(E) \cap E^{\prime}$, if $\tilde{\mu}(w) \neq \tilde{v}(w)$, then $\tilde{\eta}(w)$ is the unique point $\zeta$ on the hyperbolic circle $\rho(\zeta, \tilde{\mu}(w))=\rho(0, b(w) / 2)$ whose hyperbolic distance to $\tilde{v}(w)$ is a minimal; if $\tilde{\mu}(w)=\tilde{v}(w) \neq 0, \tilde{\eta}(w)=a \tilde{\mu}(w)$, where $a$ is a positive constant, such that $\tilde{\eta}(w)$ is on the hyperbolic circle $\rho(\zeta, \tilde{\mu}(w))=\rho(0, b(w) / 2)$; if $\tilde{\mu}(w)=\tilde{v}(w)=0$, then $\tilde{\eta}(w)=b(w)|\psi(w)| /(2 \psi(w))$. Let $H$ be a quasiconformal mapping on $R^{\prime}$ with complex dilatation $\tilde{\eta}$, and set $F_{1}=F \circ H^{-1}, G_{1}=G \circ H^{-1}$. We also denote by $\tilde{\mu}_{1}, \mu_{1}, \tilde{v}_{1}$ and $v_{1}$ the complex dilatations of $F_{1}, F_{1}^{-1}=H \circ f, G_{1}$ and $G_{1}^{-1}=H \circ g$, respectively.

Noting that

$$
\mu_{1}=\frac{\tilde{\eta}-\tilde{\mu}}{1-\tilde{\mu} \tilde{\eta}} \frac{\partial F}{\partial F} \circ f=\mu \chi_{R \backslash E}+\left(\frac{\tilde{\eta}-\tilde{\mu}}{1-\tilde{\tilde{\mu}} \tilde{\eta}} \frac{\partial F}{\overline{\partial F}} \circ f\right) \chi_{E},
$$

we conclude that

$$
\begin{aligned}
\left|\mu_{1}\right| & =|\mu| \chi_{R \backslash E}+\left|\frac{\tilde{\eta}-\tilde{\mu}}{1-\tilde{\tilde{\mu}} \tilde{\eta}} \circ f\right| \chi_{E} \\
& =|\mu| \chi_{R \backslash E}+\frac{k}{2} \chi_{\left(R \backslash F\left(E^{\prime}\right) \cap E\right.}+\frac{b(f)}{2} \chi_{F\left(E^{\prime}\right) \cap E} .
\end{aligned}
$$

So $\left|\mu_{1}\right| \geq k / 2$ on $R \backslash F\left(E^{\prime}\right)$ and $\left|\mu_{1}\right| \geq b(f) / 2$ on $F\left(E^{\prime} \backslash E_{0}^{\prime}\right)$. On the other hand, since $\left|\tilde{\mu}_{1}(H \circ f)\right|=\left|\mu_{1}\right|$, we conclude that

$$
k_{1}=k_{F_{1}}=\left\|\tilde{\mu}_{1}\left|H\left(R^{\prime}\right) \backslash H\left(E^{\prime}\right)\left\|_{\infty}=\right\| \mu_{1}\right| R \backslash F\left(E^{\prime}\right)\right\|_{\infty}=k,
$$

and

$$
\left|\tilde{\mu}_{1}\right|=\left|\tilde{\mu}_{1}\left(H \circ f \circ F \circ H^{-1}\right)\right| \leq b\left(f \circ F \circ H^{-1}\right)=b\left(H^{-1}\right) \quad \text { on } H\left(E^{\prime}\right) .
$$

Similarly,

$$
\nu_{1}=\frac{\tilde{\eta}-\tilde{v}}{1-\tilde{\tilde{v}} \tilde{\eta}} \frac{\partial G}{\overline{\partial G}} \circ g=v \chi_{G \circ f(R \backslash E)}+\left(\frac{\tilde{\eta}-\tilde{v}}{1-\tilde{\tilde{v}} \tilde{\eta}} \frac{\partial G}{\overline{\partial G}} \circ g\right) \chi_{G \circ f(E)},
$$


so we obtain by the assumption $k_{G} \leq k_{F}=k$ and the definition of $\tilde{\eta}$ that $k_{G_{1}} \leq k_{G} \leq k$, $\left|\tilde{v}_{1}\right| \leq b\left(H^{-1}\right)$ on $H\left(E^{\prime}\right)$ and hence $G_{1} \in Q\left(F_{1}, H\left(E^{\prime}\right), b\left(H^{-1}\right)\right)$.

Let $f_{1}=F_{1}^{-1}$. Then by definition,

$$
\begin{aligned}
\tau_{1} & =\tau_{F_{1}}=\mu_{1} \chi_{R \backslash F_{1}\left(H\left(E^{\prime} \backslash E_{0}^{\prime}\right)\right)}+k_{1} \mu_{1} / b\left(H^{-1} \circ f_{1}\right) \chi_{F_{1}\left(H\left(E^{\prime} \backslash E_{0}^{\prime}\right)\right)} \\
& =\mu_{1} \chi_{R \backslash F\left(E^{\prime} \backslash E_{0}^{\prime}\right)}+k \mu_{1} / b(f) \chi_{F\left(E^{\prime} \backslash E_{0}^{\prime}\right)},
\end{aligned}
$$

so $\left|\tau_{1}\right| \geq k / 2$ on $R \backslash F\left(E_{0}^{\prime}\right)=R \backslash F_{1}\left(H\left(E_{0}^{\prime}\right)\right)$. Noting that $G_{1} \circ F_{1}^{-1}=G \circ F^{-1}$, we conclude by $(5.10)$ that

$$
\iint_{R \backslash F\left(E_{0}^{\prime}\right)}\left|\frac{\alpha-\beta}{1-\bar{\alpha} \beta}\right|^{2}|\phi| \leq C_{3}\left(k,\|b\|_{\infty}\right) \iint_{R \backslash F\left(E_{0}^{\prime}\right)}\left(k|\phi|-\operatorname{Re} \tau_{1} \phi\right)
$$

for all $\phi \in Q(R)$.

Now

$$
3 \operatorname{Re} \iint_{E} \tau \phi-\operatorname{Re} \iint_{E} \tau_{1} \phi \leq(3 k / 2+k / 2) \iint_{E}|\phi|=2 k \iint_{E}|\phi| .
$$

Hence

$$
\begin{aligned}
3 \operatorname{Re} & \iint_{R \backslash F\left(E_{0}^{\prime}\right)} \tau \phi \\
& =2 \operatorname{Re} \iint_{R \backslash F\left(E_{0}^{\prime}\right) \backslash E} \tau \phi+\operatorname{Re} \iint_{R \backslash F\left(E_{0}^{\prime}\right) \backslash E} \tau \phi+3 \operatorname{Re} \iint_{E} \tau \phi \\
& \leq 2 k \iint_{R \backslash F\left(E_{0}^{\prime}\right) \backslash E}|\phi|+\operatorname{Re} \iint_{R \backslash F\left(E_{0}^{\prime}\right) \backslash E} \tau_{1} \phi+\operatorname{Re} \iint_{E} \tau_{1} \phi+2 k \iint_{E}|\phi| \\
& =2 k\|\phi\|_{R \backslash F\left(E_{0}^{\prime}\right)}+\operatorname{Re} \iint_{R \backslash F\left(E_{0}^{\prime}\right)} \tau_{1} \phi,
\end{aligned}
$$

that is,

$$
k\|\phi\|_{R \backslash F\left(E_{0}^{\prime}\right)}-\operatorname{Re} \iint_{R \backslash F\left(E_{0}^{\prime}\right)} \tau_{1} \phi \leq 3\left(k\|\phi\|_{R \backslash F\left(E_{0}^{\prime}\right)}-\operatorname{Re} \iint_{R \backslash F\left(E_{0}^{\prime}\right)} \tau \phi\right) .
$$

Finally, we obtain from (5.14) and (5.15) that

$$
\iint_{R \backslash F\left(E_{0}^{\prime}\right)}\left|\frac{\alpha-\beta}{1-\bar{\alpha} \beta}\right|^{2}|\phi| \leq 3 C_{3}\left(k,\|b\|_{\infty}\right) \iint_{R \backslash F\left(E_{0}^{\prime}\right)}(k|\phi|-\operatorname{Re} \tau \phi) .
$$

This completes the proof of Lemma 5.1.

6. Proof of Theorem 2.3. In this section we will prove Theorem 2.3, an equivalent form of Theorem 1.1. We first note the following

LEMMA 6.1. Given the class $Q\left(F, E^{\prime}, b\right)$, if $\tau_{F}$ is uniquely infinitesimally extremal in $\operatorname{Belt}\left(\tau_{F}, F\left(E_{0}^{\prime}\right)\right)$, then $F$ is uniquely extremal with respect to the extremal set $R\left(\tau_{F}\right)=\{z \in$ $\left.R ;\left|\tau_{F}(z)\right|=k_{F}\right\}$ in following sense: If $G$ is any other mapping in the class $Q\left(F, E^{\prime}, b\right)$ with $k_{G} \leq k_{F}$, then the complex dilatations of $F$ and $G$ must coincide on the set $f\left(R\left(\tau_{F}\right)\right)$. 
Proof 10. Suppose the contrary. Then there would exist some mapping $G$ in the class $Q\left(F, E^{\prime}, b\right)$ with $k_{G} \leq k_{F}$ such that the complex dilatations $\tilde{\mu}$ and $\tilde{v}$ of the mappings $F$ and $G$ satisfy the condition that $|(\tilde{\mu}(f)-\tilde{v}(f)) /(1-\overline{\tilde{\mu}(f)} \tilde{v}(f))|>\varepsilon_{0}>0$ on some compact subset $E$ of $R\left(\tau_{F}\right)$ with positive measure. By Lemma 5.1, for all $\phi \in Q(R)$, it holds that

$$
\varepsilon_{0}^{2} \iint_{E}|\phi| \leq C\left(k_{F}\|\phi\|_{R-F\left(E_{0}^{\prime}\right)}-\operatorname{Re} \iint_{R \backslash F\left(E_{0}^{\prime}\right)} \tau_{F} \phi\right) .
$$

On the other hand, since $\tau_{F}$ is uniquely infinitesimally extremal in $\operatorname{Belt}\left(\tau_{F}, F\left(E_{0}^{\prime}\right)\right)$, by our Theorem 4.2, (6.1) implies that the set $E$ has measure zero. This is a contradiction.

Now we prove Theorem 2.3. Let the class $Q\left(F, E^{\prime}, b\right)$ be given, and $\tilde{\mu}$ and $\mu$ be the complex dilatations of the mappings $F$ and $f=F^{-1}$, respectively. Suppose that $\tau=\tau_{F}$ is uniquely infinitesimally extremal in $\operatorname{Belt}\left(\tau_{F}, F\left(E_{0}^{\prime}\right)\right)$. We want to show that $F$ is uniquely extremal.

Suppose the contrary. Then there would exist some mapping $G \neq F$ in the class $Q\left(F, E^{\prime}, b\right)$ with $k_{G} \leq k_{F}$. Let $\tilde{v}$ and $v$ denote the complex dilatations of the mappings $G$ and $g=G^{-1}$, respectively. Then the set $E=\{z \in R: \tilde{v}(f) \neq \tilde{\mu}(f)\}$ has positive measure. On the other hand, Lemma 6.1 implies that $F$ is uniquely extremal with respect to $R(\tau)$, so the set $\left\{z \in E ;|\tau|=k=k_{F}\right\}$ has measure zero. Hence there exists a constant $k_{0}<k$ such that the set $\tilde{E}=\left\{z \in E ;|\tau(z)| \leq k_{0}\right\}$ has positive measure. We may assume that $\tilde{E}$ is compact.

Now we define a Beltrami differential $\tilde{\eta}$ on $R^{\prime}$ as follows: When $w \in R^{\prime} \backslash f(\tilde{E})$, $\tilde{\eta}(w)=0$; when $w \in f(\tilde{E}) \cap\left(R^{\prime} \backslash E^{\prime}\right), \tilde{\eta}(w)$ is the unique point $\zeta$ on the hyperbolic circle $\rho(\zeta, \tilde{\mu}(w))=\rho(0, k)$ whose hyperbolic distance to $\tilde{v}(w)$ is a minimal; when $w \in f(\tilde{E}) \cap E^{\prime}$, $\tilde{\eta}(w)$ is the unique point $\zeta$ on the hyperbolic circle $\rho(\zeta, \tilde{\mu}(w))=\rho(0, b(w))$ whose hyperbolic distance to $\tilde{v}(w)$ is a minimal. Let $H$ be a quasiconformal mapping on $R^{\prime}$ with complex dilatation $\tilde{\eta}$, and set $F_{1}=F \circ H^{-1}, G_{1}=G \circ H^{-1}$. We also denote by $\tilde{\mu}_{1}, \mu_{1}, \tilde{v}_{1}$ and $\nu_{1}$ the complex dilatations of $F_{1}, F_{1}^{-1}=H \circ f, G_{1}$ and $G_{1}^{-1}=H \circ g$, respectively.

Noting that

$$
\mu_{1}=\frac{\tilde{\eta}-\tilde{\mu}}{1-\overline{\tilde{\mu}} \tilde{\eta}} \frac{\partial F}{\overline{\partial F}} \circ f=\mu \chi_{R \backslash \tilde{E}}+\left(\frac{\tilde{\eta}-\tilde{\mu}}{1-\overline{\tilde{\mu}} \tilde{\eta}} \frac{\partial F}{\overline{\partial F}} \circ f\right) \chi_{\tilde{E}},
$$

we conclude that

$$
\begin{aligned}
\left|\mu_{1}\right| & =|\mu| \chi_{R \backslash \tilde{E}}+\left|\frac{\tilde{\eta}-\tilde{\mu}}{1-\overline{\tilde{\mu}} \tilde{\eta}} \circ f\right| \chi_{\tilde{E}} \\
& =|\mu| \chi_{R \backslash \tilde{E}}+k \chi_{\tilde{E} \cap\left(R \backslash F\left(E^{\prime}\right)\right)}+b(f) \chi_{\tilde{E} \cap F\left(E^{\prime}\right)} .
\end{aligned}
$$

Since $\left|\tilde{\mu}_{1}(H \circ f)\right|=\left|\mu_{1}\right|$, we conclude that $k_{1}=k_{F_{1}}=k$, and $\left|\tilde{\mu}_{1}\right| \leq b\left(H^{-1}\right)$ on $H\left(E^{\prime}\right)$.

Similarly,

$$
\nu_{1}=\frac{\tilde{\eta}-\tilde{v}}{1-\overline{\tilde{v}} \tilde{\eta}} \frac{\partial G}{\overline{\partial G}} \circ g=\nu \chi_{G \circ f(R \backslash \tilde{E})}+\left(\frac{\tilde{\eta}-\tilde{v}}{1-\overline{\tilde{v}} \tilde{\eta}} \frac{\partial G}{\overline{\partial G}} \circ g\right) \chi_{G \circ f(\tilde{E})},
$$


so we have by the assumption $k_{G} \leq k_{F}=k$ and the definition of $\tilde{\eta}$ that $k_{G_{1}} \leq k_{G} \leq k$, $\left|\tilde{v}_{1}\right| \leq b\left(H^{-1}\right)$ on $H\left(E^{\prime}\right)$ and hence $G_{1} \in Q\left(F_{1}, H\left(E^{\prime}\right), b\left(H^{-1}\right)\right)$.

Now by definition,

$$
\begin{aligned}
\tau_{1} & =\tau_{F_{1}}=\mu_{1} \chi_{R \backslash F_{1}\left(H\left(E^{\prime}\right) \backslash H\left(E_{0}^{\prime}\right)\right)}+k_{1} \mu_{1} / b\left(H^{-1} \circ f_{1}\right) \chi_{F_{1}\left(H\left(E^{\prime}\right) \backslash H\left(E_{0}^{\prime}\right)\right)} \\
& =\mu_{1} \chi_{R \backslash F\left(E^{\prime} \backslash E_{0}^{\prime}\right)}+k \mu_{1} / b(f) \chi_{F\left(E^{\prime} \backslash E_{0}^{\prime}\right)} \\
& =\tau \chi_{R \backslash \tilde{E}}+\mu_{1} \chi_{\tilde{E} \cap\left(R \backslash F\left(E^{\prime}\right)\right)}+k \mu_{1} / b(f) \chi_{\tilde{E} \cap F\left(E^{\prime}\right)} .
\end{aligned}
$$

Therefore, $\tau_{1}=\tau$ on $R \backslash \tilde{E}$, $\left\|\tau_{1}\right\|_{\infty}=\|\tau\|_{\infty}$, and $\left|\tau_{1}\right|=\left\|\tau_{1}\right\|_{\infty}$ on $\tilde{E}$. In particular, $\tau_{1}$ is an admissible variation of $\tau$ and hence is uniquely infinitesimally extremal. Then Lemma 6.1 implies that $F_{1}$ is uniquely extremal with respect to the extremal set $R\left(\tau_{1}\right)$, that is, $\tilde{\mu}_{1}\left(f_{1}\right)=$ $\tilde{v}_{1}\left(f_{1}\right)$ on $R\left(\tau_{1}\right)$. So $G_{1} \circ F_{1}^{-1}=G \circ F^{-1}$ is conformal in $\tilde{E}$ and $\tilde{v}(f)=\tilde{\mu}(f)$ on $\tilde{E}$, which is a contradiction. This completes the proof of Theorem 2.3 .

\section{REFERENCES}

[BA] A. BEURLING AND L. V. AHLFORS, The boundary correspondence under quasiconformal mappings, Acta Math. 96 (1956), 125-142

[BlmM] V. BožIn, N. Lakic, V. Marković and M. Mateljević, Unique extremality, J. Anal. Math. 75 (1998), 299-338.

[BMM] V. BoZ̆In, V. MARKović AND M. MATELJEVIĆ, Unique extremality in the tangent space of universal Teichmüller space, Integral Transform. Spec. Funct. 6 (1997), 223-227.

[EGL] C. J. EARLE, F. P. GARDINER AND N. LAKIC, Teichmüller spaces with asymptotic conformal equivalence, Inst. Hautes Études Sci. preprint 1995.

[EL] C. J. EARLE AND Z. LI, Isometrically embedded polydisks in infinite dimensional Teichmüller spaces, J. Geom. Anal. 9 (1999), 51-71.

[F] R. FEhlmann, On a fundamental variational lemma for extremal quasiconformal mappings, Comment. Math. Helv. 61 (1986), 565-580.

[FS] R. FEHLMANN AND K. SAKAN, On extremal quasiconformal mappings with varying dilatation bounds, Osaka J. Math. 23 (1986), 751-764.

[G1] F. P. GARDINER, On pratially Teichmüller Beltrami differentials, Michigan Math. J. 29 (1982), 237-247.

[G2] F. P. GARDINER, Teichmüller Theory and Quadratic Differentials, Wiley-Interscience, New York, 1987.

[GL] F. P. GARdiner AND N. LAKIC, Quasiconformal Teichmüller Theory, Math. Surveys Monogr. 76, American Mathematical Society, Providence, R.I., 2000.

[H] R. S. Hamilton, Extremal quasiconformal mappings with prescribed boundary values, Trans. Amer. Math. Soc. 138 (1969), 399-406.

[K] S. L. KRUShKal, Extremal quasiconformal mappings, Siberian Math. J. 10 (1969), 411-418.

[MM] V. MARKOVIĆ AND M. MATELJEVIĆ, The unique extremal quasiconformal mapping and uniqueness of Hahn-Banach extensions, Mat. Vesnik 48 (1996), 107-112.

[R1] E. REICH, Quasiconformal mappings with prescribed boundary values and a dilatation bound, Arch. Ration. Mech. Anal. 68 (1978), 99-112.

[R2] E. REICH, On criteria for unique extremality of Teichmüller mappings, Ann. Acad. Sci. Fenn. Ser. A I Math. 6 (1981), 289-301.

[R3] E. REICH, Extremal extensions from the circle to the disk, Quasiconformal mappings and analysis (Ann Arbor, MI, 1995), 321-335, A collection of papers honoring F. W. Gehring, Springer-Verlag, New York, Berlin Heidelberg, 1997.

[R4] E. REICH, The unique extremality counterexample, J. Anal. Math. 75 (1998), 339-347. 
[RS] E. REICH AND K. STREBEL, Extremal quasiconformal mappings with prescribed boundary values, Contributions to analysis (a collection of papers dedicated to Lipman Bers), 375-391, Academic Press, New York, 1974.

[Sa1] K. SAKAN, On extremal quasiconformal mappings compatible with Fuchsian groups, Tohoku Math. J. 34 (1982), 87-100.

[Sa2] K. SAKAN, On quasiconformal mappings compatible with a Fuchsian group, Osaka J. Math. 19 (1982), 159-170.

[Sa3] K. SAKAN, On extremal quasiconformal mappings compatible with a Fuchsian group and a dilatation bound, Tohoku Math. J. 37(1985), 79-93.

[Sa4] K. SAKAN, Necessary and sufficient conditions for extremality in certain classes of quasiconformal mappings, J. Math. Kyoto Univ. 26 (1986), 31-37.

[Sa5] K. SAKAN, A fundamental variational lemma for extremal quasiconformal mappings compatible with a Fuchsian group, Tohoku Math. J. 39 (1982), 105-114.

[Sh1] Y. Shen, On unique extremality, Complex Variables Theory Appl. 40 (1999), 149-162.

[Sh2] Y. SHEN, On Teichmüller geometry, Complex Variables Theory Appl. 44 (2001), 73-83.

[SC] Y. SHEN AND J. CHEN, Quasiconformal mappings with non-decreasable dilatations, Chinese Ann. Math. Ser. A 23 (2002), 459-466.

[St1] K. Strebel, On quasiconformal mappings of open Riemann surfaces, Comment. Math. Helv. 53 (1978), 301-321.

[St2] K. StREBel, Extremal quasiconformal mappings, Results Math. 10 (1986), 168-210.

Department of MATHEMATiCs

SUZHOU UNIVERSITY

SUZHOU 215006

P. R. CHINA

E-mail address: ylshen@suda.edu.cn 